\title{
Malignant fibrous histiocytoma-pleomorphic sarcoma, NOS gene expression, histology, and clinical course. A pilot study
}

\author{
Adrien Daigeler • Ludger Klein-Hitpass • Ingo Stricker • Oliver Müller • \\ Cornelius Kuhnen • Ansgar Michael Chromik • Lars Steinstraesser • Ole Goertz • \\ Hans-Ulrich Steinau • Marcus Lehnhardt
}

Published online: 7 March 2009

(C) Springer-Verlag 2009

\section{Erratum to: Langenbecks Arch Surg \\ DOI 10.1007/s00423-009-0465-0}

The first and family names of the authors were transposed.

The correct names are given here.

\begin{abstract}
Adrien Daigeler • Ludger Klein-Hitpass • Ingo Stricker •
Oliver Müller - Cornelius Kuhnen - Ansgar Michael Chromik • Lars Steinstraesser • Ole Goertz • Hans-Ulrich Steinau • Marcus Lehnhardt
\end{abstract}

The online version of the original article can be found at doi:10.1007/ s00423-009-0465-0.

A. Daigeler $(\varangle) \cdot$ L. Steinstraesser $\cdot$ O. Goertz $\cdot$ H.-U. Steinau $\cdot$

M. Lehnhardt

Department of Plastic Surgery, Burn Center, Hand surgery,

Sarcoma Reference Center, BG-University Hospital,

Bergmannsheil, Ruhr University Bochum,

Bürkle-de-la-Camp-Platz 1,

44789 Bochum, Germany

e-mail: adrien.daigeler@rub.de

I. Stricker · C. Kuhnen

Institute for Pathology, BG-University Hospital Bergmannsheil,

Ruhr University Bochum,

Bürkle-de-la-Camp-Platz 1,

44789 Bochum, Germany

O. Müller

Tumor Genetics Group,

Max-Planck-Institut für molekulare Physiologie,

Otto Hahnstr. 11,

44227 Dortmund, Germany

A. M. Chromik

Department of Surgery, St. Josef Hospital, Ruhr University,

Bochum, Germany

Virchowstr. 173,

45122 Essen, Germany 